Research paper

\title{
Observational study of prevalence of sleep disorder in patients with epilepsy
}

\section{Suryaprabha Turaga ${ }^{*}$, Preetika Soanpet, Jayasree Manikinda, Abhijeet Kumar Kohat, Samatha Reddy Davidi}

Department of Neurology, Nizam's Institute of Medical Sciences (NIMS), Punjagutta, Hyderabad 500082,

Andhra Pradesh, India

\section{A R T I C L E I N F O}

Article history:

Received 13 November 2015

Accepted 16 March 2016

Available online 4 May 2016

Keywords:

Sleep quality

Excessive daytime sleepiness

Epworth Sleepiness Scale (ESS) and

Pittsburgh Sleep Quality Index (PSQI)

Patient with epilepsy

\begin{abstract}
A B S T R A C T
Background: Reduced sleep quality with subsequent excessive daytime sleepiness (EDS) is found in patients with epilepsy.

Aim: To know the frequency of sleep disorders in comparison to control group to know the predictors of sleep disorders that have implications on management of patient with epilepsy.

Materials and methods: 199 patients with epilepsy and 48 controls, who attended Nizam's Institute of Medical Sciences were taken into the study.

The Epworth Sleepiness Scale (ESS) and Pittsburgh Sleep Quality Index (PSQI) are two questionnaires commonly used in clinical assessment of EDS and sleep quality.

Results: $24.6 \%$ of PWE are found to have problems with sleep disorders when compared to controls (10.6\%). PWE had significantly higher scores when compared to controls in global PSQI total scores (3.78 vs. 2.43). Sleep latency and medication use ( 0.83 vs. 0.53 and 0.52 vs. 0.06 respectively) as well as sleep quality and sleep efficiency are poor as indicated by high scores ( 0.75 vs. 0.40 and 0.22 vs. 0.20 respectively).

Conclusion: Poor sleep quality of PWE in PSQI correlated with EDS of ESS. Poor control of seizures, polytherapy, and partial epilepsy make the PWE statistically more vulnerable to poor sleep quality thereby having therapeutic implications.
\end{abstract}

(C) 2016 Indian Epilepsy Society. Published by Elsevier, a division of Reed Elsevier India, Pvt. Ltd. All rights reserved.

\section{Background}

Sleep disturbances is a common complaint and several studies have confirmed that both daytime sleepiness and poor sleep quality are common in patients with epilepsy (PWE), regardless of age. ${ }^{1,2}$ The relationship between sleep and epilepsy are complex and dynamic affecting each other in bidirectional way. Sleep can affect the expression of epilepsy by activating inter-ictal discharges and nocturnal seizures. IEDs occur most often during NREM sleep due to thalamocortical hyper synchrony. During REM sleep, seizures and IEDs become less frequent. Increased epilepsy severity, including IEDs may be associated with aggravated sleep disturbances in patients

\footnotetext{
* Corresponding author. Tel.: +91 40 9246589899/23370899; fax: +91 4023310076.

E-mail address: surmukh99@gmail.com (S.P. Turaga).

http://dx.doi.org/10.1016/j.ijep.2016.03.001

2213-6320/C 2016 Indian Epilepsy Society. Published by Elsevier, a division of Reed Elsevier India, Pvt. Ltd. All rights reserved.
} 
with epilepsy. Poor sleep quality (insufficient sleep, increased nocturnal and early morning awakenings, impaired sleep initiation) and excessive daytime sleepiness (EDS) are common complaints of patients with epilepsy (PWE). The sleep disorders and epilepsy can have an additive negative impact on quality of life, work productivity and overall health. There are not much data available in India regarding this issue.

\section{Aim}

The aims of the study are to assess the frequency of sleep disorders in PWE attending epilepsy clinic of Nizam's Institute of Medical Sciences (NIMS), a tertiary care center, in South India, as well as to evaluate the possible predisposing factors for excessive daytime sleepiness, subjective sleep quality in PWE.

\section{Materials and methods}

199 patients of the epilepsy attending epilepsy clinic of Neurology department and 48 controls who are attendants to other departments of Nizam's Institute of Medical Sciences are taken into study. Those with mental Retardation, psychiatric comorbidities, medical diseases (HTN, DM, COPD, stroke, heart diseases, sleep disorders), night work, shift work who are known to be associated with sleep disorders are excluded from study. Patients who are on medication that could affect sleep, including stimulants, antidepressants, and antipsychotic drugs are also excluded. Consent is taken from all participants of the study.

Clinical data of patients including syndromic diagnosis, age of onset, duration of illness, seizure control, aetiology of seizure, associated medical diseases, current antiepileptic drugs (AEDs) are taken into study. The Epworth Sleepiness Scale (ESS) and Pittsburgh Sleep Quality Index (PSQI) are two questionnaires commonly used in clinical assessment of EDS and Sleep quality. $\mathrm{ESS}^{3}$ is a standardized scale used for measuring sleepiness that is used to evaluate EDS in PWE. ${ }^{4,5}$ PSQI is a questionnaire that is used to evaluate overall sleep quality which includes subjective sleep quality, sleep latency, sleep duration, sleep efficiency, and sleep disturbance, medication use, and daytime dysfunction. ${ }^{6,7}$ The ESS is designed to evaluate the general level of daytime sleepiness. ${ }^{3}$ In this questionnaire, subjects are instructed to rate, on a scale of 0-3 (never $=0$, slight $=1$, moderate $=2$, high $=3$ ), the likelihood of dozing off or falling asleep in eight different situations. An ESS score $\geq 10$ was considered to be EDS. ${ }^{2,3}$ The PSQI is a 19-item self-rated questionnaire for evaluating subjective sleep quality over the previous month. ${ }^{7}$ The 19 questions are combined into seven clinically derived component scores: subjective sleep quality (C1), sleep latency (C2), sleep duration (C3), habitual sleep efficiency (C4), sleep disturbances (C5), use of sleeping medication (C6), and daytime dysfunction (C7). Each item is weighed equally, and is rated from 0 to 3 ( $0=$ no difficulty, $3=$ severe difficulty). The component scores are added to obtain a global score of 0-21, with higher scores indicating worse sleep quality.
A global sum of "5" or greater was considered to be poor overall sleep quality. ${ }^{6,7}$

\section{Statistical analysis}

Demographics are described as percentages, mean with SD. Variables between patients and controls are correlated with Fisher's exact $t$ test/chi-square test wherever appropriate. $P$ value $<0.05$ is taken as significant. As the scores of both ESS and PSQI are not normally distributed, a Mann-Whitney U test is used to explore the relationship between the groups of PWE and Spearman's correlation was used to explore the correlation between ESS and PSQI. All statistical analyses are conducted using the IBM SPSS software package (version 20).

\section{Results}

A total of 199 epilepsy patients and 48 controls are analyzed for sleep disorders. The number of Males in this study is more than that of females with the ratio being 122:77. The mean age of presentation of epilepsy patients is $25.57 \pm 11.2$ years. The most common type of presentation in our study is symptomatic focal seizures, yet the commonest type of epilepsy being chronic mesial temporal lobe epilepsy. The mean duration of illness is $9.13 \pm 8.71$ years. $73.86 \%$ (147/199) of patients with epilepsy are on polytherapy, whereas rest are on monotherapy (26.13\%) (Table 1).

$24.6 \%$ of PWE had sleep disorders, whereas $10.4 \%$ of control population had sleep disorders (Table 2). EDS which is measured as ESS $\geq 10$ is seen in $10 \%$ of PWE when compared to $6.89 \%$ in controls. ESS scores are found to be higher i.e., $4.96 \pm 3.00$ of PWE when compared to $3.60 \pm 3.54$ in controls which is statistically significant.

PWE had significantly higher scores in various aspects of sleep quality in PSQI though they are not amounting to abnormal range. PSQI total global scores are higher in PWE

Table 1 - Comparison of demographic data of PWE with and without seizures.

\begin{tabular}{llll} 
& $\begin{array}{l}\text { Controls } \\
(n=48)\end{array}$ & $\begin{array}{l}\text { Seizure free } \\
(n=69)\end{array}$ & $\begin{array}{l}\text { Non-seizure } \\
\text { free } \\
(n=130)\end{array}$ \\
\hline Age & $31.47 \pm 10.49$ & $24.51 \pm 12.14$ & $26.2 \pm 9.9$ \\
Sex (M:F) & $23: 25$ & $2: 1$ & $9: 6$ \\
Age of onset, years & & $16.67 \pm 13.97$ & $13.16 \pm 9.33$ \\
Duration, years & & $8.77 \pm 9.11$ & $7.52 \pm 8.62$ \\
Aetiology, \% & & \\
$\quad$ Cryptogenic & $33.3 \%$ & $25 \%$ \\
$\quad$ Symptomatic & $47.6 \%$ & $50 \%$ \\
$\quad$ Idiopathic & $19.04 \%$ & $25 \%$ \\
Seizure type, \% & & \\
$\quad \begin{array}{l}\text { Partial } \\
\text { Generalised }\end{array}$ & $69.2 \%$ & $40 \%$ \\
Mode of therapy, \% & $30.7 \%$ & $60 \%$ \\
$\quad$ Monotherapy & & \\
$\quad$ Polytherapy & $61.5 \%$ & $90.9 \%$ \\
\hline
\end{tabular}


Table 2 - Comparison of EPS and PSQI scores in PWE vs. controls.

\begin{tabular}{lccc} 
Sleep disorders & Controls (48) & PWE (199) & P value \\
\hline ESS & $3.60 \pm 3.54$ & $4.96 \pm 3.00$ & 0.941 \\
ESS $>$ 10 & $6.89 \%$ & $10 \%$ & \\
GLOBAL PSQI & $2.43 \pm 2.56$ & $3.78 \pm 3.19$ & 0.037 \\
PSQI > 5 & $10.4 \%$ & $24.6 \%$ & \\
Sleep latency & $0.53 \pm 0.91$ & $0.83 \pm 1.0$ & 0.033 \\
Sleep quality & $0.40 \pm 0.58$ & $0.75 \pm 0.79$ & 0.005 \\
Sleep duration & $0.35 \pm 0.57$ & $0.48 \pm 0.72$ & 0.322 \\
Sleep efficiency & $0.20 \pm 0.54$ & $0.22 \pm 0.48$ & 0.899 \\
Sleep disturbance & $0.78 \pm 0.59$ & $0.82 \pm 0.74$ & 0.983 \\
Medication use & $0.063 \pm 0.43$ & $0.52 \pm 0.54$ & 0.000 \\
Daytime dysfunction & $0.085 \pm 0.35$ & $0.44 \pm 0.76$ & 0.000 \\
\hline
\end{tabular}

when compared to controls ( $3.78 \pm 3.19$ vs. $2.43 \pm 2.56)$. Sleep latency and medication use had higher scores in PWE when compared to controls $(0.83 \pm 1.0$ vs. $0.53 \pm 0.91$ and $0.52 \pm 0.85$ vs. $0.063 \pm 0.43$ respectively). Sleep quality and sleep efficiency are poor as indicated by high scores $(0.75 \pm 0.79$ vs. $0.40 \pm 0.58$ and $0.22 \pm 0.48$ vs. $0.20 \pm 0.54$ respectively) in our study. It is found that on Spearman correlation analysis, poor sleep quality in PSQI positively correlated with excessive daytime sleepiness of ESS. Poor control of seizures and polytherapy are associated with more sleep disorders than those who do not. Patients with partial epilepsy are statistically more vulnerable to poor sleep quality (Table 3).

\section{Discussion}

The present study confirms that PWE frequently have more sleep problems (24.6\%). They have a significantly higher chance of having problems with sleep quality, sleep latency and sleep efficiency than do healthy controls. This study

\begin{tabular}{|c|c|c|c|}
\hline & PSQI < 5 (145) & PSQI > 5 (54) & $P$ value \\
\hline Age & $24.99+10.44$ & $26.65+10.22$ & 0.445 \\
\hline Sex (M:F) & 81.64 & 34.20 & 0.607 \\
\hline Aetiology, $n(\%)$ & & & 0.579 \\
\hline Symptomatic & 71 (48.9\%) & $36(66.66 \%)$ & \\
\hline Cryptogenic & 22 (15.3\%) & $11(20.37 \%)$ & \\
\hline Idiopathic & 52 (51.03\%) & 7 (22.96\%) & \\
\hline Seizure type, $n(\%)$ & & & 0.002 \\
\hline Partial & 71 (48.96\%) & $36(66.66 \%)$ & \\
\hline Generalized & 74 (51.03\%) & 19 (33.33\%) & \\
\hline $\begin{array}{l}\text { Seizure control, } \\
n(\%)\end{array}$ & & & 0.04 \\
\hline Non-seizure free & 20 & 40 & \\
\hline Seizure free & 16 & 14 & \\
\hline Age of onset & $15.66+11.40$ & $16.25+10.03$ & 0.267 \\
\hline Duration, years & $9.20+8.97$ & $8.74+8.09$ & 0.342 \\
\hline $\begin{array}{l}\text { Mode of therapy, } \\
n(\%)\end{array}$ & & & 0.002 \\
\hline Monotherapy & 35 (24.13\%) & $6(11.11 \%)$ & \\
\hline Polytherapy & $110(75.86 \%)$ & 48 (88.88\%) & \\
\hline ESS & $2.71+2.90$ & $6.26+3.95$ & 0.03 \\
\hline ESS $>10, n(\%)$ & $20(14)$ & 44 (81.48) & 0.005 \\
\hline
\end{tabular}

reveals a high percentage of PWE are with poor sleep quality, including problems with sleep quality, sleep latency, sleep duration, and sleep efficiency which is in concordance with other studies in sleep by Chen et al., etc. ${ }^{8}$ However, there was no difference between PWE and controls on the PSQI subscales of sleep disturbance and daytime dysfunction. This study suggests that EDS in PWE may be due to poor sleep quality which is in concordance with other studies in sleep by Chen et al., etc. ${ }^{8}$ In our study the lack of difference in sleep disturbance between groups which also in concordance to the study by Chen et al., etc. This may be related to the limitation of the PSQI questionnaire in detecting primary sleep disorder as sleep disturbance is occasionally related to primary sleep disorder. The mean age of presentation being very low in our study and duration of illness is very high. This is in comparison to other studies and despite young age most of them are found to be associated with sleep disorders. $73.6 \%$ of our people are having polytherapy as the most of the patients are refractory partial epilepsies which is a limitation of our study to know the individual effect of each drug. But this may also help us know the effect of polytherapy on sleep. There is increased sleep latency in our study which could be due to the multiple effects of various drugs and interaction of epilepsy with sleep. This same finding also seen in Chen et al.

However in our study, the ESS in PWE significantly correlated with PSQI, indicating that EDS in such patients is related to poor night time sleep quality. Poor sleep quality at night may lead to EDS and daytime dysfunction, which is reported in many neurologic disorders, such as epilepsy, Parkinson's disease, dementia, migraine, restless leg syndrome, etc. ${ }^{1,2,9-13}$ Poor sleep hygiene also leads to sleep fragmentation, which can exacerbate seizures and EDS in PWE. ${ }^{14}$

Polytherapy, partial seizure, and poor seizure control are important factors that contribute to poor sleep quality and lead to EDS in PWE. These findings are also similar to study by Nai-Ching et al. However, in the correlation analysis, frequent seizure attacks were associated with decreased sleep quality. Patients with partial epilepsy are more vulnerable to poor sleep quality. Patients with partial epilepsy due to different locations have different effects on epilepsy. ${ }^{1,9,15,16}$ For instance, nocturnal frontal lobe epilepsy is found to be associated with problems of sleep fragmentation and increased daytime sleepiness ${ }^{17}$ whereas temporal lobe epilepsy has been found to be associated with a high rate of sleep disruption $^{18}$ thereby contributing to poor sleep quality in PWE. Sleep is a potent activator of seizures. It activates both interictal epileptiform discharges and also seizures. ${ }^{11} \mathrm{Com}$ pared to wakefulness, the spike rate is increased during nonrapid eye movement sleep and is reduced during rapid eye movement sleep. ${ }^{19}$ Furthermore, frequent seizure attacks may influence sleep quality. ${ }^{11}$ Increased epilepsy severity, including increased interictal epileptiform activity, may be associated with aggravated sleep disturbances in PWE. ${ }^{20}$ The present study demonstrates that patients with poor seizure control have poorer sleep quality than those with fewer seizure attacks, indicating that seizure severity may also play a crucial role in sleep problems.

It is notable that different AEDs have different effects on sleep. Phenobarbital and benzodiazepines have been shown to alter normal sleep architecture. ${ }^{21}$ Phenytoin and valproic acid 
disrupt sleep while gabapentin improves sleep quality. ${ }^{22}$ The effect of carbamazepine on sleep is controversial, ${ }^{22}$ whereas topiramate, lamotrigine, and levetiracetam reportedly have no effect on sleepiness. ${ }^{20}$

\section{Conclusions}

$24.6 \%$ of patients with epilepsy had sleep disorders. Poor nighttime sleep quality may lead to EDS and daytime dysfunction. Poor seizure control, partial epilepsy and polytherapy are closely related to sleep disorders. These relationships have their implications in providing best management in sleep disturbances.

\section{Limitations}

The influence of specific drugs cannot be determined from the present study, because of insufficient case numbers, further studies are needed to clarify the issue.

\section{Conflicts of interest}

The authors have none to declare.

\section{REFEREN C E S}

1. Maganti R, Hausman N, Koehn M, Sandok E, Glurich I, Mukesh BN. Excessive daytime sleepiness and sleep complaints among children with epilepsy. Epilepsy Behav. 2006;8(February (1)):272-277.

2. Piperidou C, Karlovasitou A, Triantafyllou N, et al. Influence of sleep disturbance on quality of life of patients with epilepsy. Seizure. 2008;17(October (7)):588-594.

3. Johns MW. A new method for measuring daytime sleepiness: the Epworth sleepiness scale. Sleep. 1991;14 (December (6)):540-545.

4. Manni R, Tartara A. Evaluation of sleepiness in epilepsy. Clin Neurophysiol. 2000;111(September):S111-S114.

5. Malow BA, Bowes RJ, Lin X. Predictors of sleepiness in epilepsy patients. Sleep. 1997;20(December (12)):1105-1110.

6. Knutson KL, Rathouz PJ, Yan LL, Liu K, Lauderdale DS. Stability of the Pittsburgh Sleep Quality Index and the Epworth Sleepiness Questionnaires over 1 year in early middle-aged adults: the CARDIA study. Sleep. 2006;29 (November (11)):1503-1506.
7. Buysse DJ, Hall ML, Strollo PJ, et al. Relationships between the Pittsburgh Sleep Quality Index (PSQI), Epworth Sleepiness Scale (ESS), and clinical/polysomnographic measures in a community sample. J Clin Sleep Med. 2008;4 (December (6)):563-571.

8. Chen N-C, Tsai M-H, Chang C-C, et al. Sleep quality and daytime sleepiness in patients with epilepsy. Acta Neurol Taiwan. 2011;20(December (4)):249-256.

9. Foldvary-Schaefer N. Sleep complaints and epilepsy: the role of seizures, antiepileptic drugs and sleep disorders. J Clin Neurophysiol. 2002;19:514-521.

10. Comella CL. Sleep disturbances and excessive daytime sleepiness in Parkinson disease: an overview. J Neural Transm Suppl. 2006;349-355.

11. Kotagal P, Yardi N. The relationship between sleep and epilepsy. Semin Pediatr Neurol. 2008;15:42-49.

12. Boddy F, Rowan EN, Lett D, O'Brien JT, McKeith IG, Burn DJ. Subjectively reported sleep quality and excessive daytime somnolence in Parkinson's disease with and without dementia, dementia with Lewy bodies and Alzheimer's disease. Int J Geriatr Psychiatry. 2007;22:529-535.

13. Seidel S, Hartl T, Weber M, et al. Quality of sleep, fatigue and daytime sleepiness in migraine - a controlled study. Cephalalgia. 2009;29:662-669.

14. Foldvary N. Sleep and epilepsy. Curr Treat Options Neurol. 2002;4:129-135.

15. de Weerd A, de Haas S, Otte A, et al. Subjective sleep disturbance in patients with partial epilepsy: a questionnaire based study on prevalence and impact on quality of life. Epilepsia. 2004;45:1397-1404.

16. Vignatelli L, Bisulli F, Naldi I, et al. Excessive daytime sleepiness and subjective sleep quality in patients with nocturnal frontal lobe epilepsy: a case-control study. Epilepsia. 2006;47:73-77.

17. Zucconi M, Oldani A, Smirne S, Ferini-Strambi L. The macrostructure and microstructure of sleep in patients with autosomal dominant nocturnal frontal lobe epilepsy. J Clin Neurophysiol. 2000;17:77-86.

18. Crespel A, Baldy-Moulinier M, Coubes P. The relationship between sleep and epilepsy in frontal and temporal lobe epilepsies: practical and physiopathologic considerations. Epilepsia. 1998;39:150-157.

19. Sammaritano M, Gigli GL, Gotman J. Interictal spiking during wakefulness and sleep and the localization of foci in temporal lobe epilepsy. Neurology. 1991;41:290-297.

20. Bell C, Vanderlinden H, Hiersemenzel R, Otoul C, Nutt D, Wilson S. The effects of levetiracetam on objective and subjective sleep parameters in healthy volunteers and patients with partial epilepsy. J Sleep Res. 2002;11: 255-263.

21. Declerck AC, Wauquier A. Influence of antiepileptic drugs on sleep patterns. Epilepsy Res Suppl. 1991;2:153-163.

22. Legros B, Bazil CW. Effects of antiepileptic drugs on sleep architecture: a pilot study. Sleep Med. 2003;4:51-55. 